\title{
Spiking with recombinant allergens to improve allergen extracts: benefits and limitations for the use in routine diagnostics
}

\section{Part 19 of the Series Molecular Allergology}

\author{
Johannes Huss-MarP' ${ }^{1}$, Monika RaUlF ${ }^{2}$, ThILO JAKOB ${ }^{1}$
}

${ }^{1}$ Allergy Research Group, Department of Dermatology, University Medical Center Freiburg, Germany; ${ }^{2}$ Institute for Prevention and Occupational Medicine of the German Social Accident Insurance, Institute of the Ruhr University Bochum, Germany (IPA)

Key words Spiking molecular allergy diagnostics latex - hazelnut wasp venom bee venom recombinant

\section{Submitted}

April 8, 2015

\section{Accepted}

April 21, 2015

\section{German version} www.springermedizin.de/ allergo-journal

\section{Abstract}

Essentially, allergen components offer three possibilities to improve in vitro IgE diagnostics:

a) Allergen components can be used individually for IgE determination.

b) Allergen components can be combined as a mix in one test.

c) Individual allergen components can be specifically added to the extract.

Option (a) is currently being used most extensively in practice, while (b) represents more of a theoretical possibility. The specific addition ("spiking") of allergen components to an allergy extract (c) has been performed in the past for the ImmunoCAP ${ }^{\circledast}$ tests for latex (09/2001), hazelnut (05/2006) and wasp venom (06/2012). Through this approach under-represented allergen components could be compensated and the analytical sensitivity of the test systems significantly increased. In combined use with molecular Singleplex tests, these modified tests allow for new diagnostic possibilities. Clear communication from the manufacturer regarding in which test and from what time point on, recombinant allergens were added - and where this was not done despite under-represented allergen compo- nents - is important for the interpretation of the test results in routine clinical practice.

Cite this as Huss-Marp J, Raulf M, Jakob T. Spiking with recombinant allergens to improve allergen extracts: benefits and limitations for the use in routine diagnostics. Part 19 of the Series Molecular Allergology. Allergo J Int 2015;24:236-43

DOI: 10.1007/s40629-015-0058-0

$\begin{array}{ll}\text { Abbrevations } \\ \text { CCD: } & \text { Cross-reactive carbohydrate determinants } \\ \text { lgE: } & \text { Immunoglobulin E } \\ \text { nsLTP: } & \text { Non-specific lipid transfer protein } \\ \text { NPV: } & \text { Negative predictive value } \\ \text { PPV: } & \text { Positive predictive value } \\ \text { slgE: } & \text { Specific immunoglobulin E } \\ \text { WV } & \text { Wasp venom }\end{array}$




\section{Introduction}

Molecular allergy diagnostics is based on the insight that not the entire allergen source, but the isolated allergens within are relevant for sensitization and clinical manifestation of the allergic reaction. Essentially, the use of allergen components in diagnostics offers three possibilities of modifying an in vitro(immunoglobulin E) IgE Singleplex test:

a) allergen components can be used individually for IgE determination,

b) the available allergen components can be combined as mix in one test to replace a natural extract or

c) individual allergen components can be specifically added to the extract (Fig. 1).

While option (a) currently represents the most extensively used application of molecular allergy diagnostics, option (b) is rarely used in practice, since this procedure is elaborate, expensive and the benefits are questionable. The last-mentioned application (c), so-called "spiking" of an extract with allergen components with the goal of increasing test sensitivity is, however, used in some common, extract-based in vitro tests. This procedure is particularly useful if allergen components are under-represented in the conventional extract-based test.

There are a number of reasons why one or several allergen components are under-represented in an extract-based test. They range from the composition and variation of the natural source material with regard to the occurrence of allergen components, the extractability of the allergen components from the raw material to stability of the components after extraction. Test-specific aspects such as, for example, the bonding behaviors of allergen components, etc. also play a role.

Since important allergen components were under-represented in the conventional IgE tests for latex, hazelnut and wasp venom and the tests were modified in the past by "spiking", we will present examples of this procedure from the areas of latex, hazelnut and wasp venom allergy and discuss them with regard to their clinical implications.

\section{Improvement of diagnostics through allergen addition in latex allergy}

The type I latex allergy represents a classical, IgE mediated, immediate-type reaction. The proteins in the natural latex milk of the pará rubber tree Hevea brasiliensis are the triggers of latex allergy. Currently eighteen latex allergens (including isoforms) have been identified and described as Hev b 1 to Hev b 15 according to the IUIS allergen nomenclature (Hev b is derived from Hevea brasiliensis; www.allergen. org) [1].

Since the skin test extracts to detect a type I latex allergy are increasingly no longer commercially available, the serological test for latex-specific IgE not only represents an additional, but in the meantime almost the only method to detect sensitization to latex.

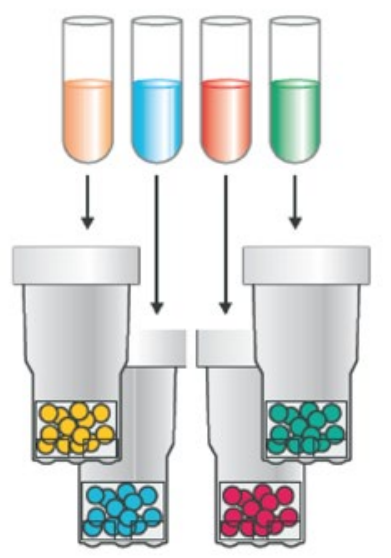

a

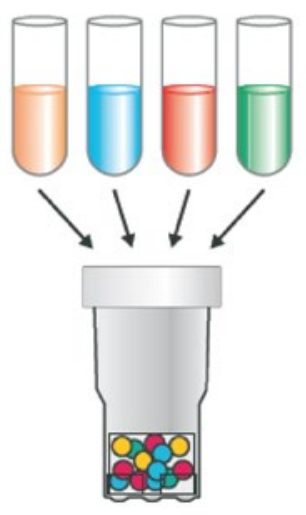

b

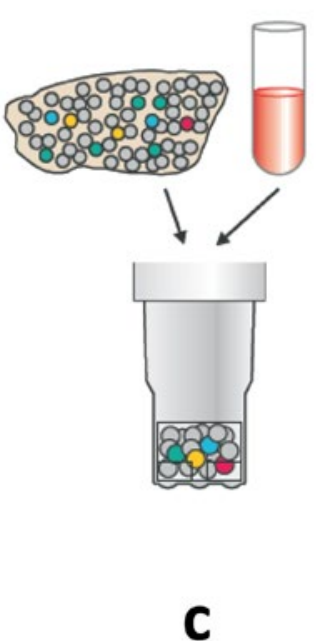

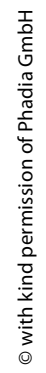

Fig. 1: Use of allergen components in allergy diagnostics: a: Allergen components used individually, b: Allergen components combined as mix in one test, c: "Spiking" of an allergenic extract with individual allergen components. 


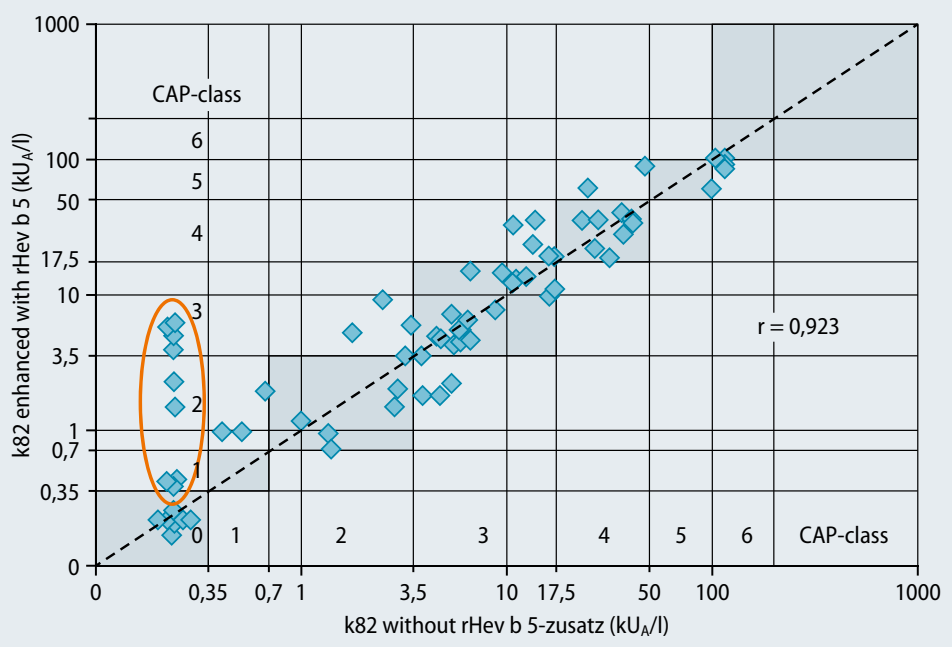

Fig. 2: Comparison of latex ImmunoCAP ${ }^{\circledR}$-results für k82 without rHev b 5 und with k82 with rHev b 5. Study collective: Health care workers with latex allergy, which were tested with both tests mentioned above $(n=68)$. (Modified according to [6])

As investigations by Chen et al. [2] or Lundberg et al. [3] showed, 16 of 111 latex patients from the health care system with a positive latex skin test reaction and the clinical symptoms of a latex allergy tested negative in the specific IgE test with the commonly used latex allergen extract. By the use of recombinant individual latex allergens it was possible to show, that 8 of these patients were monosensitized to Hev b 5 . $\mathrm{Hev} b 5$ is an acidic protein that is very similar to a protein in kiwi fruit and is considered a major latex allergen along with Hev b 1 and Hev b 6.01/6.02. It is recognized by patients in the health care system allergic to latex as well as patients with spina bifida at a comparable rate $[4,5]$. Only the addition of rHev b 5 to the allergen material usually used for detecting latex-specific IgE and the preparation of a new ImmunoCAP ("k82 supplemented with rHev b 5"; [3]) led to relevant improvement in the test sensitivity - particularly in individual cases (Fig. 2). These results demonstrate a new strategy for the manufacturing of standardized allergy diagnostics: if relevant allergens are too unstable to withstand the production steps of standardized allergen extracts or if they are missing, stable recombinant proteins can be added during production. " $\mathrm{k} 82$ plus $\mathrm{rHev} \mathrm{b} 5$ " has been commercially available since spring 2002 in order to improve the in vitro latex diagnostics. This new ImmunoCAP ${ }^{\circ}$ replaced the original ImmunoCAP ${ }^{\circledR} \mathrm{k} 82$ without be- ing identified as " $\mathrm{k} 82$ plus $\mathrm{rHev} \mathrm{b} 5$ ". It continued to be identified as k82. As Tab. 1 shows, the sensitivity of the latex-specific IgE determination was increased from $76 \%$ (latex extract without rHev b 5) to $90 \%$ with a test efficiency of $93.75 \%$ using the ImmunoCAP supplemented with $\mathrm{rHev} b$ 5. The use of an ImmunoCAP ${ }^{\oplus}$ manufactured for research purpose, consisting of the latex allergens $\mathrm{rHev} b 1, \mathrm{rHev} b 5$, rHev 6.01 and $\mathrm{rHev} b 8$, improved test efficiency compared to the ImmunoCAP ${ }^{\circledR}$ without rHev b 5 from $86.7 \%$ to $90.6 \%$ (Tab. 1), but ultimately did not achieve the efficiency of the rHev b 5-supplemented "spiked" ImmunoCAP ${ }^{\circledR}[6]$.

\section{Benefits and disadvantages of allergen addition in hazelnut allergy}

Hazelnuts are among the most common triggers of food allergies worldwide. Associated symptoms range from the oral allergy syndrome to severe systemic and even fatal reactions. To date, a number of allergen components of the hazelnut have been identified, which can be categorized into the protein families of PR-10 proteins (Cor a 1), profilins (Cor a 2), non-specific lipid transfer proteins (nsLTP; Cor a 8), storage proteins (Cor a 9, Cor a 11, Cor a 14) and oleosins (Cor a 12, Cor a 13) (www. allergen.org).

In this case, as well as in a number of other food allergies, the molecular sensitization profile allows a risk assessment regarding the patient's hazard potential upon allergen exposure. Among other things, this depends on the amount of allergen in the allergenic source as well as on its stability, e.g. to heat and digestion. Storage proteins and nsLTPs are often associated with an increased risk potential, whereas PR-10 protein sensitizations are frequently associated with birch pollen and suggest cross-reactivity. For example, sensitization to the heat-stable and digestion-resistant nsLTP or storage proteins of hazelnut, are frequently accompanied by severe systemic symptoms. In contrast, the heat-labile hazelnut component Cor a 1 (PR-10 protein) is usually a trigger of mild allergic reactions, such as the oral allergy syndrome [7]. Thus, Cor a 8, Cor a 9 and Cor a 14 are particularly important for estimation of the risk potential in patients with hazelnut allergy [7].

These allergen components were already sufficiently represented in the past in the extract-based IgE test that allowed for recognition of these patients. However, the PR-10 protein Cor a 1 that particularly stands for cross-reactivity to birch pollen was not well represented in the extract. This fact led to clinical studies with the hazelnut ImmunoCAP ${ }^{\circ}$ f17 (Thermo Fisher Scientific, Freiburg, Germany) sometimes only having a low sensitivity for the test, depending on the patient collective and geographical region. For example, a study from the Nether- 


\begin{tabular}{|c|c|c|c|c|c|}
\hline Method & Sensitivity (\%) & Specinty (\%) & PPV (\%) & NPV (\%) & Efficacy (\%) \\
\hline k82 "alt" & $/ 6,0$ & 98,3 & 98,1 & 18,1 & 86,1 \\
\hline k82 + rHev b 5 & 90,0 & 98,3 & 98,4 & 89,4 & 93,8 \\
\hline rHev-b-Mix* & 83,6 & 98,3 & 98,2 & 84,3 & 90,6 \\
\hline
\end{tabular}

lands only detected sensitization in 18 of 31 patients (58\%) with confirmed hazelnut allergy using f17 (f17 sIgE $\geq 0.35 \mathrm{KU} / \mathrm{l}$ ) [8]. These results and comparable ones in further studies led the manufacturer to make the decision to modify the ImmunoCAP f17 by "spiking" it, with the goal of increasing sensitivity. The investigations performed in this context were published [9] and ultimately led to a significant increase in test sensitivity: The "old" ImmunoCAP f17 did not detect 8 patients with confirmed hazelnut allergy in a collective of 50 patients from Central Europe, while the new f17 test supplemented with recombinant Cor a 1 detected sensitization to hazelnut in all patients. This corresponds to an increase in sensitivity from $84 \%$ to $100 \%$ [9]. The Cor a 1-supplemented $\mathrm{f} 17$ test was introduced to the market in May 2006 and it subsequently replaced the test used previously.

The response from allergists to this change was not only positive as shown by a publication from Sicherer et al. [10]: Many pediatricians in the US had previously used the $\mathrm{f} 17$ test primarily in the diagnosis of hazelnut allergies in infancy and childhood which can generally be attributed to storage proteins. The hazelnut extract supplemented with Cor a 1 now no longer only detected sensitizations to nsLTP and storage proteins, but also PR-10 proteins with high sensitivity. These sensitizations are partly clinically irrelevant and can usually be attributed to cross-reactions due to tree pollen allergy. It was particularly criticized that with the new Cor a 1-supplemented f17 ImmunoCAP ${ }^{\circledR}$ differentiation between different sensitization patterns was no longer possible, and that this change was not sufficiently communicated by the manufacturer.

Today, Cor a 1, Cor a 8, Cor a 9 and Cor a 14 are available as molecular Singleplex tests in addition to the Cor a 1-supplemented hazelnut ImmunoCAP f17; they allow for detailed recognition of the patient's sensitization profile and for the implementation of molecular-based allergy diagnostics in patients with hazelnut allergy.

\section{Improvement of test sensitivity by allergen addition in wasp venom allergy}

Another example of improved diagnostics by addition of a recombinant individual allergen to the allergen extract can be found in wasp venom allergy. The serological IgE diagnostics of hymenoptera venom allergy is complicated by a high degree of cross-reactivity between bee and wasp venom extracts. For example, up to $45 \%$ of our patients exhibited dual sensitization to both insect venoms [11]. This cross-reactivity is either caused by cross-reactive carbohydrate determinants (CCD) or is based on protein homology between individual allergens in bee and wasp venom. The introduction of CCDfree species-specific marker allergens (Api m 1 for bee venom or Ves $\mathrm{v} 5$ and Ves $\mathrm{v} 1$ for wasp venom) that allow for definite differentiation between bee and wasp venom allergy, significantly improved the serological diagnostics of hymenoptera venom allergy [11, 12].

The first report on the benefits of rApi $\mathrm{m} 1$ and rVes $v 5$ in the IgE diagnostics of hymenoptera venom allergy already stated that positive sIgE levels to the marker allergen rVes v 5 (i209), but negative IgE serology to wasp venom (ImmunoCAP i3) were measured in 5 of 7 patients with a clear history of anaphylaxis after a wasp sting [11]. A larger follow-up examination of 308 patients with wasp venom allergy confirmed these initial findings [13]. Here, only $83.4 \%$ of the patients showed sensitization to the wasp venom (i3), while IgE sensitization ( $\geq 0.35 \mathrm{kUA} / \mathrm{L})$ was detectable in $96 \%$ of the cases using the individual allergens Ves v 1 and Ves v 5. Among patients with wasp venom allergy who tested negative regarding IgE against wasp venom extract (i3) IgE could be detected against rVes v 5 in $84.4 \%(42 / 51, \geq 0,35 \mathrm{kUA} / \mathrm{l})$ of patients. Comparative examinations on IgE reac- 

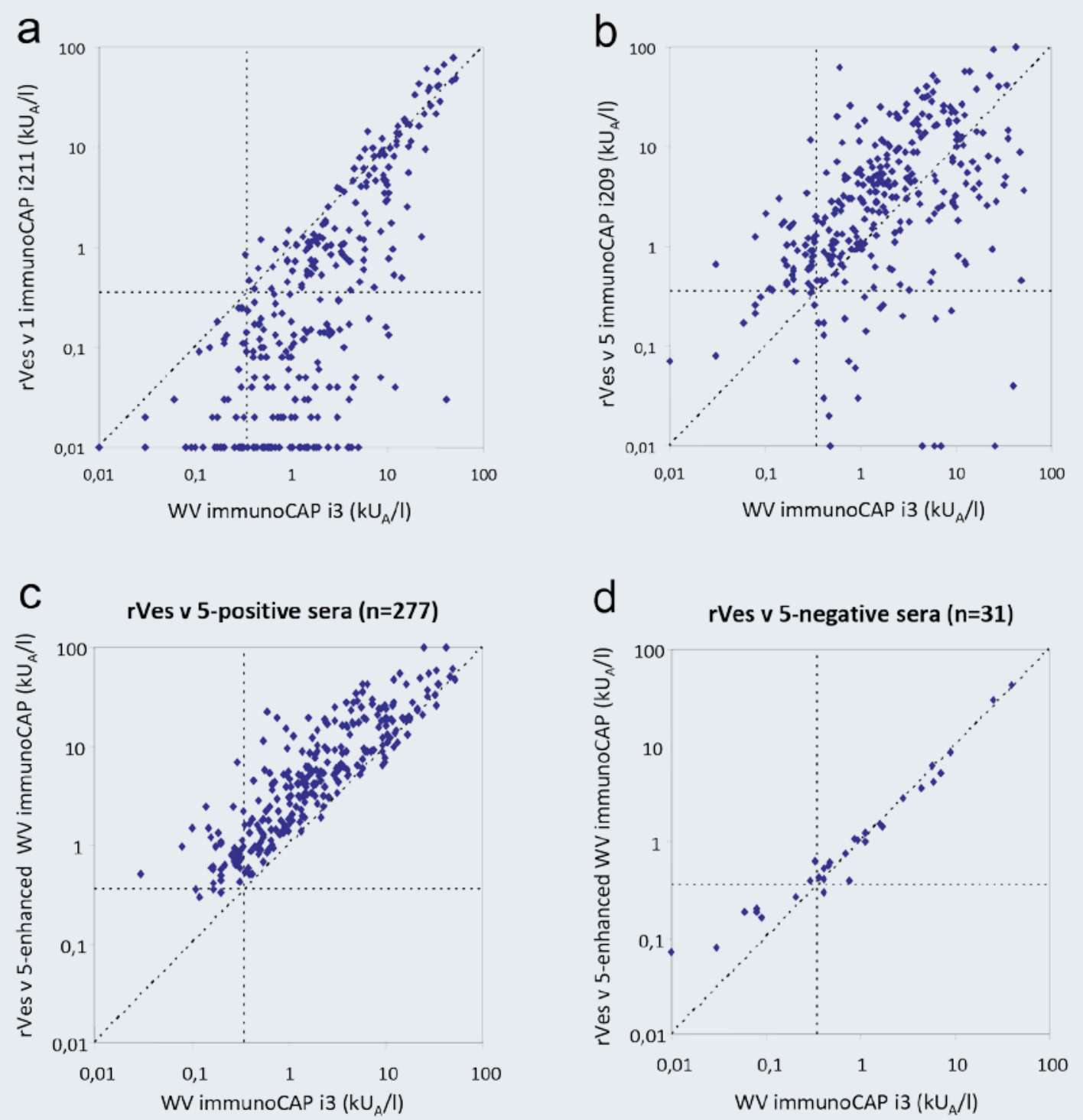

Fig. 3: Comparison of IgE- reactivity against wasp venom extract (i3), rVes v 1 (i211), rVes v 5 und Ves v 5-spiked WV in patients with wasp venom allergy. a: Comparison of IgE-levels to WV (i3) against rVes v 1 ( $n=308)$, b: Comparison of IgE-levels to WV (i3) against rVes v $5(\mathrm{n}=308)$, c: Comparison of IgE-levels to WV (i3) against rVes v 5-enhanced WV in Ves v5-positive patients $(n=277)$, d: Comparison of IgE-levels to WV (i3) against rVes v 5-enhanced WV in Ves v-5negative patients $(n=31)$. Dotted horizontal and vertical lines indicate cutoff values $\geq 0,35 \mathrm{kUA} / \mathrm{l}$; dotted diagonal line corresponds to a relationship of $1: 1$. (Adapted from [13]; with kind permission of Elsevier)

tivity to wasp venom extract (i3) and rVes v 1 (i211) detected higher values for the total venom than for the individual allergen in almost all patients which suggests that only part of the IgE reactivity is directed against the selected allergen (Fig. 3a). In contrast, comparative examinations on IgE reactivity to wasp venom extract (i3) and rVes v 5 (i209) detected IgE reactivity to the individual allergen that was on average 2.4 times higher than that to whole venom (Fig. 3b). This observation suggested that the IgE immunoreactivity to Ves $\mathrm{v} 5$ is under-represented in the wasp venom extract (i3). 
Various mechanisms can theoretically be responsible for a phenomenon of this type, such as, for example

a) a lack of the allergen Ves $v 5$ in wasp venom extract,

b) poor or insufficient coupling of Ves $v 5$ in natural wasp venom to the solid phase of the test system,

c) steric blockade of the IgE epitopes to Ves v 5 by endogenous inhibitors, amongst others.

This apparently absent, IgE immunoreactivity in the conventional ImmunoCAP ${ }^{\circledR} \mathrm{i} 3$, was compensated by "spiking" the wasp venom with recombinant Ves v 5 [13]. A direct comparison with the previous wasp venom ImmunoCAP ${ }^{\circledR}$ in Ves $\mathrm{v} 5$ positive patients detected significantly elevated $\mathrm{IgE}$ reactivity for the rVes v 5-supplemented ImmunoCAP (Fig. $3 c$ ). Both CAP variants delivered comparable results in Ves $v$ 5-negative patients. In comparison to the previous wasp venom ImmunoCAP ${ }^{\circledR}$, the Ves $\mathrm{v}$ 5-supplemented ImmunoCAP ${ }^{\circledR}$ captured $96.8 \%$ of the patients allergic to wasp venom. The test sensitivity increased from $83.4 \%$ to $96.8 \%$ through addition of rVes v 5 (Fig. 4). Similar results were also reported from other groups [14]. The observed increase in the sensitivity was not associated with reduced specificity of the test system. Based on this data, rVes v 5-supplemented wasp venom was introduced for routine diagnostics in June 2012. After a transitional period the previous (not Ves v 5-supplemented) wasp venom Immuno$\mathrm{CAP}^{\oplus}$ (i3) was taken off the market.

Unfortunately the manufacturer failed to adequately communicate this change in the test system and provide the different variants with individual names. This is particularly relevant for follow-up observations in the context of specific immunotherapy. On the whole, it can be presumed that before 2012, sIgE to wasp venom (i3) was measured with the non-supplemented ImmunoCAPs, while all values collected from 2013 onwards were analyzed with the new rVes v 5-supplemented ImmunoCAP ${ }^{\circledast}$.

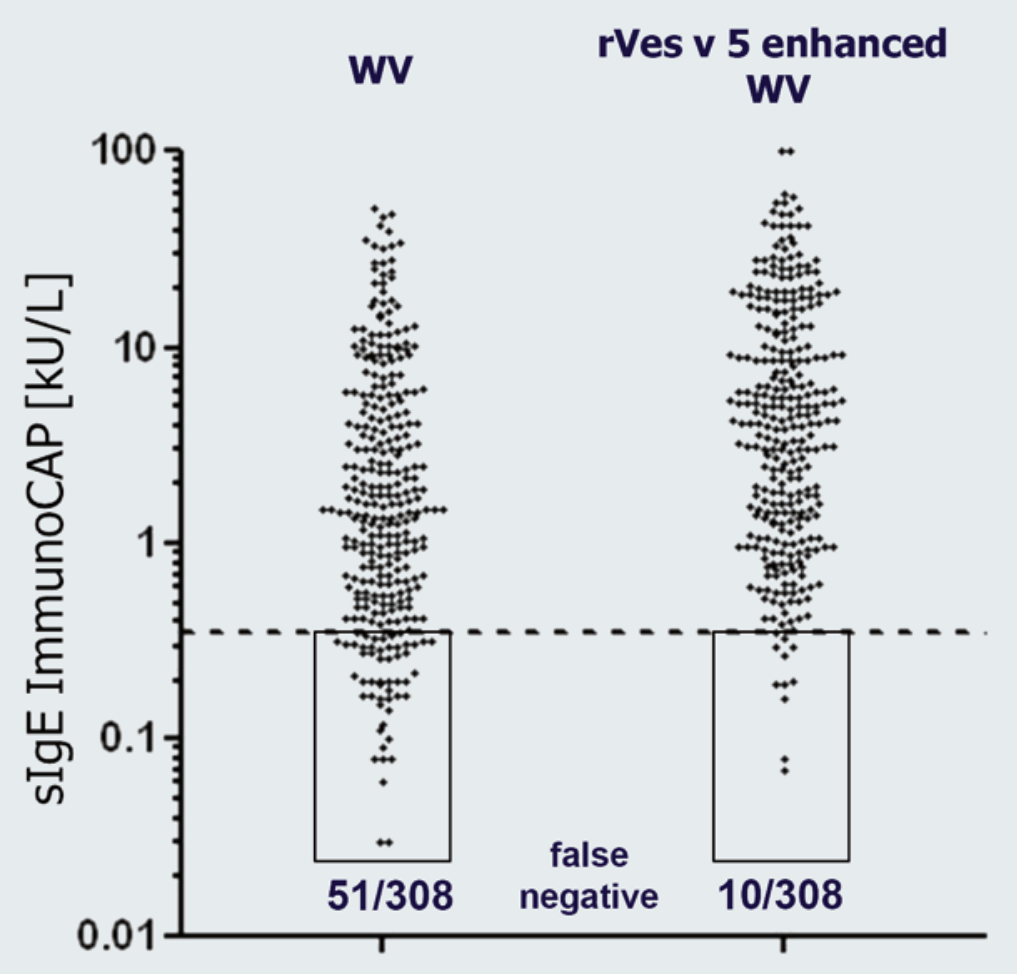

\section{Sensitivity $\quad 83,4 \% \quad \longrightarrow 96,8 \%$}

Fig. 4: Enhancement of dignostic sensitivity in wasp venom allergy by spiking with $r$ Ves $v$ 5. slgE-reactivity against WV (i3) without (left) and with addition of rVes v 5 (right) in 308 patients with WV allergy. (adapted from [13]) 
The significant improvement in sensitivity resulting from the addition of rVes $\mathrm{v} 5$ suggests that further individual allergens such as, e.g. Ves $\mathrm{v} 1$, Ves $\mathrm{v} 2$ or Ves $\mathrm{v} 3$ could possibly be used for improvement of test performance. However, this is not the case, as investigations on the sera of patients with a clear history of wasp venom allergy, but without sIgE to Ves v 5-supplemented wasp venom showed [15]. The same goes for the individual diagnostic bee venom allergens Api m 1, Api m 2, Api m 3, Api m 4, Api $m 5$ and Api $\mathrm{m} \mathrm{10}$, that have been characterized to date [16]. In patients with a clear history of bee venom allergy but without positive sIgE values to whole bee venom extract, the application of Api m 1, Api m 2, Api m 3, Api m 4, Api $m 5$ and Api m 10 did not lead to an improvement in diagnostic sensitivity [15].

\section{Additional value of molecular diagnostics and conclusion for routine clinical practice}

The examples of addition of recombinant individual allergens to extract-based tests listed in this chapter show the potential of this approach for improved diagnosis, but also the associated problems. While the sensitivity of the tests was significantly increased for latex, hazelnut and wasp venom, the example of the hazelnut ImmunoCAP ${ }^{\star} \mathrm{f} 17$ shows that the diagnostic discriminatory power can certainly also be reduced as a result. Today this problem can be overcome by combining further allergen component-based Singleplex tests. Based on the broad spectrum of available molecular tests a detailed sensitization profile can be obtained which, taken together with history and clinical findings, allows diagnosis and risk assessment. The decision to alter an extract-based in vitro allergy test by adding individual recombinant allergens should always be carefully considered, since the performance and application of the test can be permanently affected all over the world. Not all extract-based IgE tests where allergen components are under-represented were supplemented in the past by addition of the relevant allergens. For example, the allergen component Tri a 19 (omega-5-gliadin) is under-represented in the wheat ImmunoCAP ${ }^{\circledR}$ and Gly $\mathrm{m} 4$ in the soy ImmunoCAP ${ }^{\circledast}$ but no decision to add these to the extracts in recombinant form was made, since the allergen components are available as molecular Singleplex tests and thus there is no diagnostic gap.

Finally, it should be noted that molecular-based allergy diagnostics opens new diagnostic possibilities in allergy through the option of "spiking" in combination with molecular Singleplex tests. Clear communication on the part of the manufacturer, to which test recombinant allergens were added and from what time point on - and also where this was not done despite under-represented allergen components - is essential in order to allow clini- cians to optimally utilize these possibilities for improved patient management.

Prof. Dr. Johannes Huss-Marp, MD, MBA

Allergy Research Group, Department of Dermatology, University Medical Center Freiburg, Germany current address: AbbVie Deutschland GmbH \& Co. KG Mainzer Straße 81, 65189 Wiesbaden, Germany

E-Mail: johannes.huss-marp@abbvie.com

\section{Conflict of interest}

Thilo Jakob has received research grants from Phadia $\mathrm{GmbH} /$ Thermo Fisher Scientific, Freiburg and Fooke Laboratories, Neus, additional he received fees as lecturer and advisor from Phadia GmbH/Thermo Fisher Scientific, Freiburg. Johannes Huss-Marp was Medical Director at Phadia GmbH/Thermo Fisher Scientific, Freiburg, from 2009-2014.

\section{Cite this as}

Huss-Marp J, Raulf M, Jakob T. Spiking with recombinant allergens to improve allergen extracts: benefits and limitations for the use in routine diagnostics. Part 19 of the Series Molecular Allergology. Allergo J Int 2015;24: 236-43 DOI: 10.1007/s40629-015-0058-0

\section{References}

1. Raulf-Heimsoth M, Rihs HP. Latexallergene: Sensibilisierungsquellen und Einzelallergenprofile erkennen. Allergo J 2011;20:241-3

2. Chen Z, Rihs HP, Slater JE, Paupore EJ, Schneider EM, Baur $X$. The absence of Hev b 5 in capture antigen may cause false-negative results in serologic assays for latex-specific IgE antibodies. J Allergy Clin Immunol 2000;105:S8

3. Lundberg M, Chen Z, Rihs HP, Wrangsjö K. Recombinant spiked allergen extract. Allergy 2001;56:794-5

4. Akasawa A, Hsieh LS, Martin BM, Liu T, Lin Y. A novel acidic allergen, Hev $b 5$, in latex. Purification, cloning and characterization. J Biol Chem 1996;271:25389-93

5. Slater JE, Vedvick T, Arthur-Smith A, Trybul DE, Kekwick RGO. Identification, cloning, and sequence of a major allergen (Hev b 5) from natural rubber latex (Hevea brasiliensis). J Biol Chem 1996;271:25394-9

6. Raulf-Heimsoth M, Rihs HP, Rozynek P, Cremer R, Gaspar Â, Pires $G$ et al. Quantitative analysis of IgE reactivity profiles in patients allergic or sensitized to natural rubber latex (Hevea brasiliensis). Clin Exp Allergy 2007;37:1657-67

7. Masthoff LJ, Mattsson L, Zuidmeer-Jongejan L, Lidholm J, Andersson K, Akkerdaas JH et al. Sensitization to Cor a 9 and Cor a 14 is highly specific for a hazelnut allergy with objective symptoms in Dutch children and adults. J Allergy Clin Immunol 2013;132:393-9

8. Wensing M, Penninks AH, Hefle SL, Akkerdaas JH, Ree R van, Koppelman SJ et al. The range of minimum provoking doses in hazelnut-allergic patients as determined by double-blind, placebocontrolled food challenges. Clin Exp Allergy 2002;32:1757-62

9. Andersson K, Ballmer-Weber BK, Cistero-Bahima A Östling J, Lauer I, Vieths S, Lidholm J. Enhancement of hazelnut extract for IgE testing by recombinant allergen spiking. Allergy 2007;62:897-904

10. Sicherer SH, Dhillon G, Laughery KA, Hamilton RG, Wood RA. Caution: The Phadia hazelnut ImmunoCAP (f17) has been supplemented with recombinant Cor a 1 and now detects Bet v 1-specific IgE, which leads to elevated values for persons with birch pollen allergy. J Allergy Clin Immunol 2008;122:413-4 
11. Hofmann SC, Pfender N, Weckesser S, Huss-Marp J, Jakob T. Added value of IgE detection to $\mathrm{rApi} \mathrm{m} 1$ and $\mathrm{rVes} v 5$ in patients with Hymenoptera venom allergy. J Allergy Clin Immunol 2011;127:265-7

12. Müller $U$, Schmid-Grendelmeier $P$, Hausmann $O$, Helbling A. IgE to recombinant allergens Api $m 1$, Ves $\vee 1$, and Ves $v$ 5 distinguish double sensitization from crossreaction in venom allergy. Allergy 2012;67:1069-73

13. Vos B, Köhler J, Müller S, Stretz E, Ruëff F, Jakob T. Spiking venom with rVes $v 5$ improves sensitivity of IgE detection in patients with allergy to Vespula venom. J Allergy Clin Immunol 2013;131:1225-7
14. Ebo DG, Faber M, Sabato V, Leysen J, Bridts CH, De Clerck LS. Component-resolved diagnosis of wasp (yellow jacket) venom allergy. Clin Exp Allergy 2013;43:255-61

15. Rafei-Shamsabadi D, Müller S, Pfützner W, Spillner E, Rueff F, Jakob T. Recombinant allergens rarely allow identification of Hymenoptera venom allergic patients with negative specific IgE to whole venom preparations. J Allergy Clin Immunol 2014;134:493-4

16. Köhler J, Blank S, Müller S, Frick M, Bantleon F, Huss-Marp $J$ et al. Component resolution reveals additional major allergens in bee venom allergic patients. J Allergy Clin Immunol 2014;133:1383-9 